\title{
Comparison of Metal and Metal Oxide Media for Phosphopeptide Enrichment Prior to Mass Spectrometric Analyses
}

\author{
Matthew B. Gates, Kenneth B. Tomer, and Leesa J. Deterding \\ Laboratory of Structural Biology, National Institute of Environmental Health Sciences, National Institutes of \\ Health, Research Triangle Park, North Carolina, USA
}

\begin{abstract}
Several affinity resins consisting of ionic metals or metal oxides were investigated for their phosphopeptide enrichment capabilities with subsequent mass spectrometric analyses. Commercially-available enrichment metal oxide affinity chromatography (MOAC) resins using manufacturer's and/or published protocols were compared and evaluated for the most efficient and selective method that could be implemented as a standard enrichment procedure. From these comparative analyses, using a tryptic digest of casein proteins, it was determined that in our hands, two of the resins out-performed the others based on a variety of criteria, including the number of phosphorylation sites identified during MS analyses, the lower numbers of nonspecifically bound peptides observed, and the limits of detection. Applicability of these enrichment resins to a complex biological mixture was investigated. For this work, a mixture of avian histones was digested, subjected to titanium dioxide phosphopeptide enrichment, and analyzed by mass spectrometry. Eight phosphorylated tryptic peptides were observed following enrichment and subsequent LC/MS/MS analyses. Of note, seven of the eight phosphopeptides were not observed without titanium dioxide enrichment. From these analyses, four sites of phosphorylation were unequivocally determined, two of which have not been reported previously. Four additional phosphopeptides were observed; however, the site of phosphorylation could not be distinguished but was localized to one of two possible amino acids. These methods should aid in the investigation of proteins post-translationally modified with phosphate, especially those present at low concentrations as was demonstrated by successful enrichment at the femtomole level. (J Am Soc Mass Spectrom 2010, 21, 1649-1659) Published by Elsevier Inc. on behalf of American Society for Mass Spectrometry
\end{abstract}

$\mathrm{O}$ ne of the most common and important means of biologically regulating protein activity is through reversible phosphorylation and dephosphorylation. Phosphorylation is involved in the regulation of gene expression and protein synthesis, which controls cell growth, division, or differentiation. To better understand the molecular basis of these regulatory mechanisms, it is useful to identify the specific amino acid residues undergoing phosphorylation.

A number of different approaches to the identification of phosphorylation sites are commonly used, however, mass spectrometry is emerging as the technique of choice [1-3]. Even with recent advances in mass spectrometry instrumentation, the detection and determination of sites of phosphorylation remains a challenge since both the amount of phosphorylated protein as well as the stoichiometry of phosphorylation is often times present at low relative abundance. In addition, the suppression of low-level phosphopeptides in com-

Address reprint requests to Dr. L. Deterding, Laboratory of Structural Biology, National Institute of Environmental Health Sciences (NIEHS), National Institutes of Health, P.O. Box 12233, MD F0-03, Research Triangle Park, NC 27709, USA. E-mail: deterdi2@niehs.nih.gov plex mixtures and the low sensitivity of phosphorylated peptides under positive ionization conditions due to the negative charges on the phosphate moiety can be difficult obstacles to overcome when analyzing phosphopeptides and proteins by mass spectrometry.

In recent years, the development of enrichment techniques, such as immobilized metal ion affinity chromatography (IMAC) and metal oxide affinity chromatography (MOAC), before mass spectrometric analyses has proven beneficial for the isolation of phosphopeptides from mixtures with high specificity and for the determination of sites of phosphorylation (reviewed in [3-6]). The phosphopeptides can be analyzed by MS following elution from the enrichment resin or can be directly analyzed by MALDI/MS without prior elution [3-10].

Of these enrichment approaches, the metal oxides (e.g., titanium dioxide, zirconium dioxide) show great promise for enhanced selectivity of phosphopeptides. A variety of buffer conditions and/or chemical modification of the phosphate group have been employed to enhance the selectivity of the metal oxide enrichment media [3, 5, 6, 11-23]. For example, 
it has been shown that inclusion of organic displacers (e.g., 2,5-dihydroxybenzoic acid) during binding minimizes the level of nonspecifically bound peptides [13]. In addition, the methyl esterification of peptides before enrichment has been shown to enhance selectivity of the MOAC resins [19].

The goal of the present study is to evaluate several commercially-available enrichment MOAC resins using manufacturer's and/or published protocols for the most efficient and selective method that could be implemented as a standard enrichment procedure for complex biological samples. A titanium dioxide compact reaction column (CRC) packed with Titansphere $\mathrm{TiO}_{2}$ resin, NuTips comprised of either $\mathrm{TiO}_{2}, \mathrm{ZrO}_{2}$, or a $\mathrm{TiO}_{2} / \mathrm{ZrO}_{2}$ mixture, as well as magnetic $\mathrm{TiO}_{2}$ beads (Phos-Trap Kit) were evaluated and compared with one another as well as with our use of an Fe-nitrilotriacetic acid (Fe-NTA) IMAC CRC [7-10]. The parameters used to compare different enrichment methods were based on their selectivity for phosphopeptides, robustness, and reproducibility. In addition, the limits of detection and the applicability of the metal oxides to enrich phosphopeptides from a complex biological sample consisting of core and linker histones from chicken erythrocytes were investigated.

\section{Materials and Methods}

Ethylenediaminetetraacetic acid (EDTA), $\alpha$-cyano-4hydroxycinnamic acid ( $\alpha$-cyano), 2,5-dihydroxybenzoic acid (DHB), ammonium bicarbonate (ABC), formic acid (FA), bovine $\alpha$-casein, and $\beta$-casein (from bovine milk) were purchased from Sigma-Aldrich Chemical Company (St. Louis, MO, USA). The $\alpha$-cyano was recrystallized from hot methanol and stored in the dark at room temperature; $18 \mathrm{M} \Omega$ water was prepared on a model RO 40 water system (Hydro Service and Supplies, Durham, NC, USA). Acetonitrile (ACN) and acetic acid were purchased from Caledon (Georgetown, Ontario, Canada). Trifluoroacetic acid (TFA) was acquired from Pierce (Rockford, IL, USA), phosphoric acid (PA) from EMD Chemicals (Gibbstown, NJ, USA), and ethanol (EtOH) from Warner-Graham (Cockeysville, MD, USA). All solvents were HPLC grade. Ferric chloride $\left(\mathrm{FeCl}_{3}\right)$ was purchased from Allied Chemical (Brighton, UK). Ni-NTA resin was purchased from Qiagen (Valencia, CA, USA). Compact reaction columns (CRC) and filters (10 $\mu \mathrm{m}$ pore size) were obtained from USB Corporation (Cleveland, OH, USA). Gallus histones were purchased from Upstate Biotechnology (Lake Placid, NY, USA).

\section{Tryptic Digestion Conditions}

The proteins were subjected to digestion with sequencinggrade modified porcine trypsin (Promega Corporation, Madison, WI, USA). Enzyme was added at a protein: enzyme ratio of 20:1 and digests were allowed to proceed for at least two hours at $37^{\circ} \mathrm{C}$. For the comparison of the enrichment studies, one microgram each of $\alpha$ - and $\beta$ - casein was loaded onto the resins. Two independent sets of digests were prepared for the enrichment studies.

\section{Iron NTA IMAC CRC}

Fe-NTA IMAC columns were prepared as previously reported [7-10].

\section{Titanium Dioxide CRC}

The $\mathrm{TiO}_{2}$ MOAC procedure was adopted from Larsen et al. [13]. Briefly, $1 \mathrm{mg}$ of Titansphere $\mathrm{TiO}_{2}$ resin $(10 \mu \mathrm{m}$ particle size) was added to $10 \mu \mathrm{L}$ resin buffer $(80 \%$ ACN $, 19.9 \% \mathrm{H}_{2} \mathrm{O}, 0.1 \%$ TFA) and loaded into a CRC tube. Titansphere $\mathrm{TiO}_{2}$ resin was a gift from GL Sciences (Torrance, CA, USA). Binding of phosphopeptides to the $\mathrm{TiO}_{2}$ column was achieved by first draining the buffer, then loading the protein digest mixture with $20 \mu \mathrm{L}$ protein buffer $(100 \mathrm{mg} / \mathrm{mL}$ DHB in $80 \% \mathrm{ACN}$, $19.9 \% \mathrm{H}_{2} \mathrm{O}$, and $0.1 \% \mathrm{TFA}$ ). The mixture was allowed to incubate at room temperature for $10 \mathrm{~min}$, after which, the column was drained, and washed, $1 \times$ with $30 \mu \mathrm{L}$ protein buffer and $1 \times$ with $30 \mu \mathrm{L}$ resin buffer. Elution of the bound peptides was accomplished by adding 5 $\mu \mathrm{L}$ of $200 \mathrm{mM}$ ABC solution ( $\mathrm{pH}$ 10.5) to the column and incubated for $5 \mathrm{~min}$ at room temperature. The $5 \mu \mathrm{L}$ eluent was then drained and diluted with $5 \mu \mathrm{L}$ water. $0.5 \mu \mathrm{L}$ of the eluent solution was spotted on a MALDI target with $0.5 \mu \mathrm{L}$ of MALDI matrix (either a saturated solution of $\alpha$-cyano in 45:45:10 ethanol:water:FA or 20 $\mathrm{mg} / \mathrm{mL} \mathrm{DHB}$ in $\left.50 \% \mathrm{ACN}, 49 \% \mathrm{H}_{2} \mathrm{O}, 1 \% \mathrm{PA}\right)$.

\section{Phos-Trap Kit}

The Phos-Trap magnetic titanium beads, binding buffer, and washing buffer (buffers included in kit) were prepared according to the manufacturer's instructions (Perkin Elmer, Waltham, MA, USA). Briefly, a 10 $\mu \mathrm{L}$ aliquot of magnetic beads was diluted to $200 \mu \mathrm{L}$ in de-ionized water and placed in a 96-well plate. The beads were washed $3 \times$ with $200 \mu \mathrm{L}$ binding buffer. After placing the plate on a separation magnet, which moved the beads to an inner o-ring of the 96-well plate, the supernatant was removed. The sample was diluted 1:10 in binding buffer and incubated with beads for 10 min. Three $200 \mu \mathrm{L}$ washes of binding buffer were followed by a single wash of $100 \mu \mathrm{L}$ washing buffer. The peptides bound to the magnetic beads were eluted with $20 \mu \mathrm{L}$ of elution buffer, lyophilized, and resuspended in $1 \%$ FA just before MS analyses.

\section{Glygen NuTips}

Enrichment using Glygen Corporation's (Columbia, MD, USA) NuTips was performed similarly to the titanium CRC, with minor variations. The tips, which consist of either titanium dioxide, zirconium dioxide, or a mixture of both, were first equilibrated by washing the resin $10 \times$ with $5 \mu \mathrm{L}$ of binding solution $(80 \% \mathrm{ACN}$, 
$15 \% \mathrm{H}_{2} \mathrm{O}, 5 \%$ TFA, $\mathrm{pH}<3$ ) [13], after which excess buffer was aspirated from the tip. Before loading, the digest sample was mixed 1:1 with binding solution. This mixture was loaded onto the tip by taking $2.5 \mu \mathrm{L}$ aliquots and aspirating and expelling each aliquot $\sim 20$ times over several minutes. This was repeated until the desired amount of digest was loaded. Washes consisting of $80 \% \mathrm{ACN}, 19 \% \mathrm{H}_{2} \mathrm{O}, 1 \%$ TFA $(\mathrm{pH}<3)$ followed $(10 \times 5 \mu \mathrm{L})$, and the bound peptides were eluted with $10 \mu \mathrm{L}$ of elution solution $\left(2 \% \mathrm{NH}_{4} \mathrm{OH}\right.$ in water, $\mathrm{pH}=$ 11). To prevent loss of the phosphate moieties under highly basic conditions, the eluent was acidified with 2 $\mu$ L FA.

\section{Mass Spectrometry}

LC/ESI/MS/MS analyses were performed using an Agilent (Santa Clara, CA, USA) 1100 nanoHPLC interfaced with an Agilent XCT Ultra Ion Trap (IT) equipped with an HPLC Chip Cube MS interface or a Waters Q-TOF Premier hybrid mass spectrometer equipped with a nanoAcquity UPLC system (Manchester, UK). Automated data dependent acquisitions were employed using either CID or ETD fragmentation mechanisms. ETD of parent ions was accomplished using a fluoranthene source for the production of radical anions with a $40 \mathrm{~ms}$ accumulation time. Positive ion MALDI/MS analyses were performed using either a Voyager Super DE-STR (Applied Biosystems, Framingham, MA, USA) or a Waters Micro MX (Waters Corp., Beverly, MA, USA). Four independent enrichments and subsequent MALDI/MS analyses were performed on all MOAC resins. Additional details regarding the MS analyses are in the Supplementary Material, which can be found in the electronic version of this article.

\section{Results and Discussion}

Commercially-available $\alpha$-casein is a mixture of the S1 variant (major component), which has nine known sites of phosphorylation and the S2 variant (minor component) with 11 known sites of phosphorylation. $\beta$-casein contains five sites of phosphorylation. The $\alpha$ - and $\beta$-casein tryptic digests were mixed together in solution and analyzed by MALDI/MS (Supplemental Figure S1). The spectrum contains abundant proteolytic peptides, however, no casein phosphopeptides were observed in this analysis.

\section{MALDI/MS Analyses}

Fe-NTA IMAC. We have previously shown that phosphopeptides can be directly analyzed by MALDI/MS without prior elution from immobilized metal ion affinity media (IMAC) [7-10] as elution of phosphopeptides from this media can be inefficient $[8,10]$. Figure 1a shows the direct on-resin MALDI-TOF mass spectrum of a casein digest mixture after Fe-NTA bead enrichment. Ions corresponding in mass to several phos- phopeptides are observed. Specifically, ions corresponding in mass to eight protonated phosphopeptides representing eight sites of phosphorylation from $\alpha_{\mathrm{S} 1}$, nine from $\alpha_{\mathrm{S} 2}$, and one from $\beta$-casein are observed (Table 1). Additionally, two oxidized phosphopeptides $\left(\alpha_{\mathrm{S} 1}\right.$ T16 and $\alpha_{\mathrm{S} 1}$ T7) were also observed; however, we did not determine whether or not oxidation preceded binding to the resin. Surprisingly, ions corresponding to peptides containing four $\left(\alpha_{\mathrm{S} 2} \mathrm{~T} 2\right.$ and $\left.\alpha_{\mathrm{S} 2} \mathrm{~T} 7\right)$ and five $\left(\alpha_{\mathrm{S} 1} \mathrm{~T} 8\right)$ phosphate groups were observed. Typically, under positive ion conditions, the MS analysis of peptides containing multiple phosphorylation sites is difficult $[24,25]$. Although at least five nonphosphorylated peptides were observed with the Fe-NTA enrichment (Figure 1a, ions labeled with a filled circle), the abundance of the nonspecifically bound peptides is relatively low ( $<8 \%$ relative abundance). The ion of $\mathrm{m} / \mathrm{z}$ 1832.83 (labeled with an asterisk in Figure 1a) has been previously reported by Larsen et al. as being a phosphorylated sequence variant and may be attributed to alternative splicing [13]. The Fe-NTA resin was capable of enriching phosphopeptides representing 18 of 25 known sites of phosphorylation from a casein digest mixture (Figure 1a and Table 1).

Titanium dioxide resin. In 2004, the selectivity of titanium dioxide for phosphopeptides was first reported [12]. To investigate the utility, in our hands, of titanium dioxide for phosphopeptide enrichment, casein digest mixtures were loaded onto CRCs packed with a commercially-available $\mathrm{TiO}_{2}$ resin (GL Sciences), eluted, and the resulting MALDI mass spectra obtained. A representative MALDI spectrum from these enrichments is shown in Figure 1b. In this spectrum, ions corresponding in mass to eight unique protonated tryptic phosphopeptides as well as two oxidized forms were observed. These peptides represent 14 unique sites of phosphorylation. Only two nonspecifically bound peptides were observed (T13 and T28-30 from the S2 variant of $\alpha$-casein) with this resin. As was observed with the Fe-NTA IMAC resin (Figure 1a), peptides containing four or five phosphate groups were observed. Interestingly, the level to which the Fe IMAC or $\mathrm{TiO}_{2}$ MOAC resins enriched various peptides differed, thereby indicating a difference in specificity of binding between the Fe-NTA and titanium resins or elution from the $\mathrm{TiO}_{2}$ resin. For example, the monophosphorylated $\beta$-casein T6 peptide is observed at ca. $80 \%$ relative abundance in the MALDI spectrum from the $\mathrm{TiO}_{2}$ resin whereas this peptide is observed at ca. $10 \%$ relative abundance in the MALDI spectrum from the Fe IMAC resin. Conversely, the doubly phosphorylated $\alpha$-casein S1 T7 peptide is observed at $\sim 60 \%$ relative abundance versus $5 \%$ relative abundance in the MALDI spectra from the $\mathrm{TiO}_{2}$ resin versus the IMAC resin. The MALDI/MS results of four independent $\mathrm{TiO}_{2}$ enrichments from two independent digestion mixtures are summarized in Table 1 . The number of times a specific site of phosphorylation was observed by MALDI/MS 
(a)
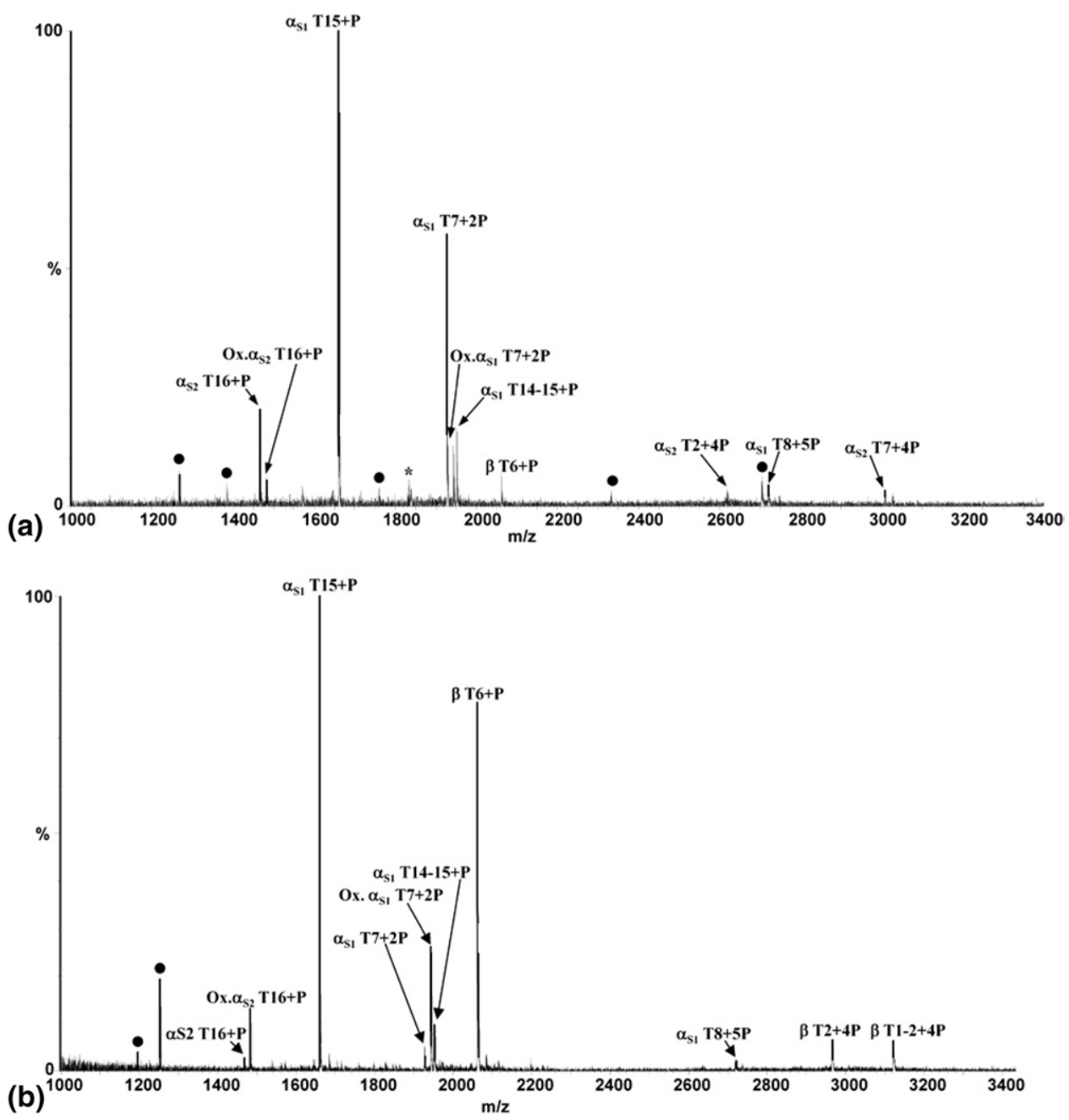

Figure 1. MALDI/MS of an $\alpha$ - and $\beta$-casein tryptic digest following (a) Fe-NTA IMAC CRC enrichment, and (b) titanium dioxide (GL Sciences) MOAC CRC enrichment. The ion labeled with an asterisk corresponds in mass to a previously reported monophosphorylated casein variant [13]. Ions labeled with filled circles represent nonspecifically bound peptides.

from the four independent MOAC enrichments is indicated in the table. Similar to previous reports that organic additives (e.g., DHB and citric acid) can be used to displace nonspecifically bound peptides $[13,14,16]$, we observed that the selectivity of the titanium resin was improved using DHB in the loading solution. As was reported by Larsen et al. [13], we also found that $100 \mathrm{mg} / \mathrm{mL}$ DHB was optimal for the casein digest mixture.

Prepacked MOAC enrichment tips. Commercially-available prepacked products (Glygen NuTips) were also employed to determine their applicability for enrichment of phosphopeptides. These products contain three different resins (titanium dioxide, zirconium dioxide, or a mixture of both) and were evaluated using the casein digest mixtures. First, the utility of the $\mathrm{TiO}_{2} \mathrm{NuTip}$ was evaluated. A representative MALDI/MS spectrum of the casein phosphopeptides enriched using the $\mathrm{TiO}_{2}$ NuTip (Figure 2a) shows ions which correspond in mass to 14 phosphopeptides and represents 21 unique sites of phosphorylation. Moreover, only two nonspecifically bound nonphosphorylated peptides (Figure 2a, ions labeled with a filled circle) of relatively low abundance (i.e., $<5 \%$ ) were observed in the eluent from this resin (Figure 2a). The results of four independent enrichments using this resin are shown in Table 1. Interestingly, a comparison of the data obtained from the $\mathrm{TiO}_{2}$ CRCs versus the $\mathrm{TiO}_{2}$ NuTips shows a higher number of unique sites of phosphorylation (8 versus 22) observed with the $\mathrm{TiO}_{2}$ NuTips in four of four enrichments. In addition, the peptides that contain three or more phosphate groups are observed more reproducibly with the $\mathrm{TiO}_{2}$ NuTip than with the $\mathrm{TiO}_{2}$ CRCs. If one includes the phosphopeptides that were observed in three of four independent enrichments, all known sites of phosphorylation were enriched using the $\mathrm{TiO}_{2}$ NuTip protocol.

Similarly, a representative MALDI-TOF mass spectrum (Figure $2 \mathrm{~b}$ ) acquired from the eluent following 


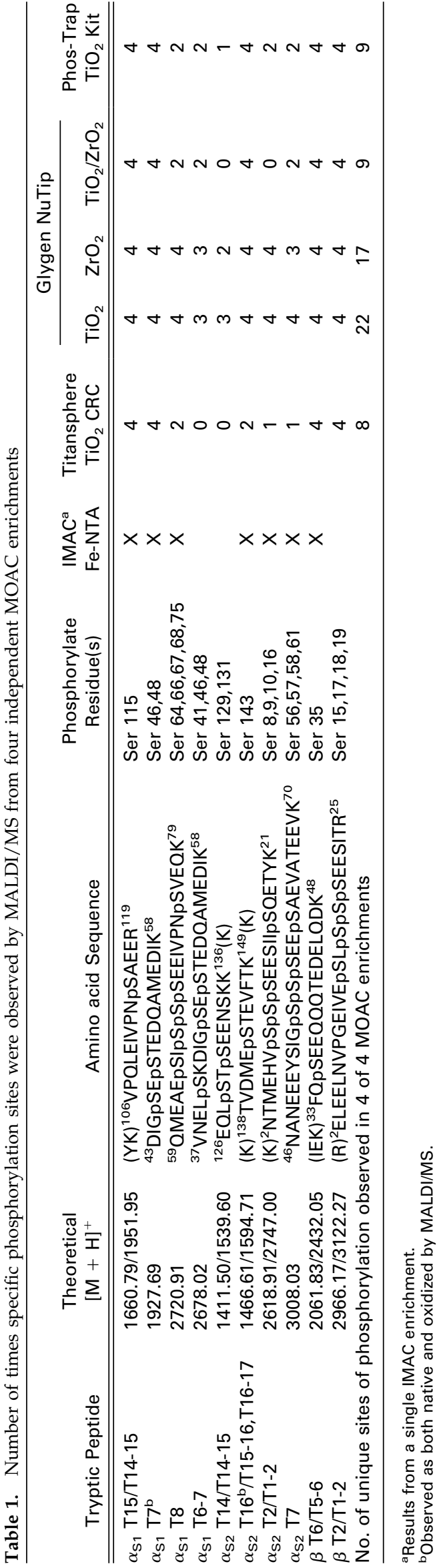

enrichment with Glygen's $\mathrm{ZrO}_{2}$ tip shows ions corresponding in mass to 10 unique phosphopeptides representing 17 sites of phosphorylation. The number of unique sites of phosphorylation observed from four independent enrichments using the $\mathrm{ZrO}_{2}$ resin is 17 (Table 1). Moreover, many nonphosphorylated casein peptides (e.g., $\alpha_{\mathrm{S} 1} \mathrm{~T} 10, \mathrm{~T} 17$, and $\beta$ T10-11) are observed using the $\mathrm{ZrO}_{2}$ resin (Figure $2 \mathrm{~b}$, ions labeled with filled circles), thereby, indicating greater nonspecific binding compared with either the $\mathrm{TiO}_{2}$ NuTips or the Titansphere $\mathrm{TiO}_{2}$ resin.

Combining different metal oxide resins in one tip has potential for improved recovery of phosphopeptides as well as enhanced selectivity. It has also been reported that zirconium dioxide is more selective for singly phosphorylated peptides and titanium dioxide for multiphosphorylated peptides $[15,16]$. Therefore, a mixed resin tip consisting of titanium dioxide and zirconium dioxide was evaluated using the casein digest mixtures. The MALDI mass spectrum acquired from one eluent from the mixed resin shows eleven ions corresponding in mass to phosphopeptides representing 15 unique sites of phosphorylation from the digest mixture (Figure 2c). From these data, the number of nonspecifically bound peptides observed is lower than with the $\mathrm{ZrO}_{2}$ resin alone and are more similar to the titanium dioxide NuTips. MALDI/MS analyses from four independent mixed resin enrichments are summarized in Table 1. The number of unique sites of phosphorylation observed with the mixed $\mathrm{TiO}_{2} / \mathrm{ZrO}_{2}$ resin NuTip in four of four enrichments is similar to that obtained with the $\mathrm{TiO}_{2}$ CRCs (nine and eight, respectively) and is lower than either the single $\mathrm{TiO}_{2}$ resin NuTip (i.e., 22) or the single $\mathrm{ZrO}_{2}$ resin NuTip (i.e., 17). Also of note, in our hands, the mixed resin tips would often leak resin out of the tip when high $\mathrm{pH}$ buffers were used, thereby, complicating their analyses. Perhaps this is one reason why the number of unique sites of phosphorylation observed with the mixed resin tips was lower than for either the $\mathrm{TiO}_{2}$ or $\mathrm{ZrO}_{2}$ resin by itself.

It has been reported that the enrichment capabilities of a given resin can vary based on the manner of its manufacture, the method by which it is affixed to the pipette tip, as well as the buffer conditions used during loading and washing steps. Therefore, we investigated the affect of the buffer conditions with the NuTips. Three sets of conditions were tested: (1) binding buffer of $3.3 \%$ formic acid and washed with water [15]; (2) binding buffer of $80 \% \mathrm{ACN}, 15 \% \mathrm{H}_{2} \mathrm{O}, 5 \%$ TFA) [16] followed by water washes; and (3) binding buffer of $80 \%$ ACN, $15 \% \mathrm{H}_{2} \mathrm{O}, 5 \%$ TFA followed by a wash solution of $80 \% \mathrm{ACN}, 19 \% \mathrm{H}_{2} \mathrm{O}, 1 \%$ TFA [16]. The use of binding and washing system 3 resulted in the observation of much higher phosphopeptide selectivity (e.g., less than five nonspecifically bound peptides observed) and was the protocol used for the analyses shown in Figure 3. Although increased selectivity was observed for the Titansphere titanium dioxide resin when the organic displacer DHB was used, the addition of this 

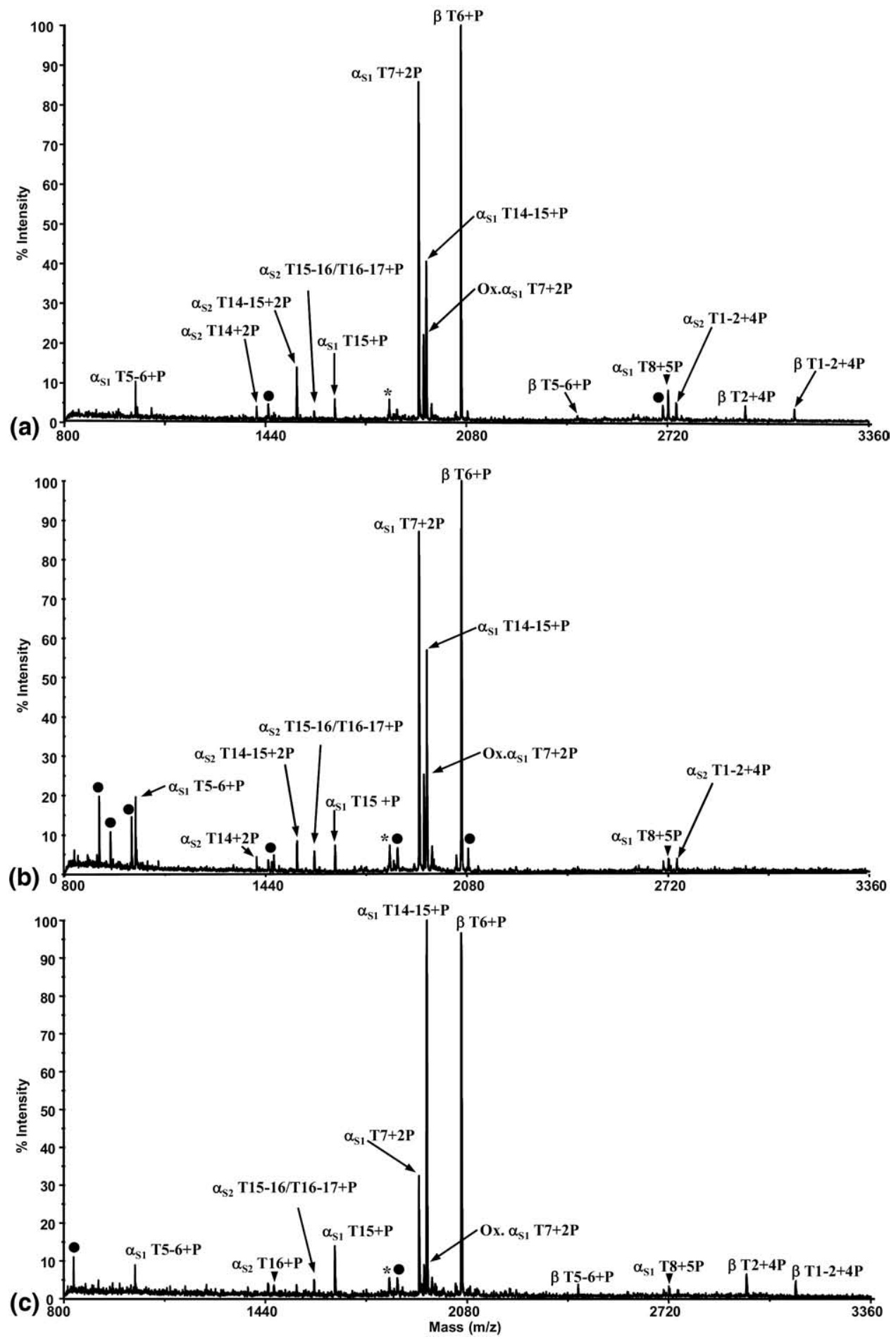

Figure 2. MALDI mass spectra of an $\alpha$ - and $\beta$-casein tryptic digest resulting from (a) titanium dioxide NuTip enrichment, (b) zirconium dioxide NuTip enrichment, and (c) a mixed resin tip enrichment consisting of zirconium and titanium dioxide. The ion labeled with an asterisk corresponds in mass to a previously reported monophosphorylated casein variant [13]. Ions labeled with filled circles represent nonspecifically bound peptides.

modifier did not significantly improve the enrichment capabilities of the NuTips in terms of the number of phosphopeptides observed.

To summarize, more unique sites of phosphorylation were reproducibly obtained using titanium dioxide enrichment NuTips than either the zirconium dioxide NuTips or the mixed resin NuTips (Table 1). In addition, the number of nonspecifically bound peptides observed with the zirconium dioxide NuTip was, generally, greater in comparison with that of the titanium 


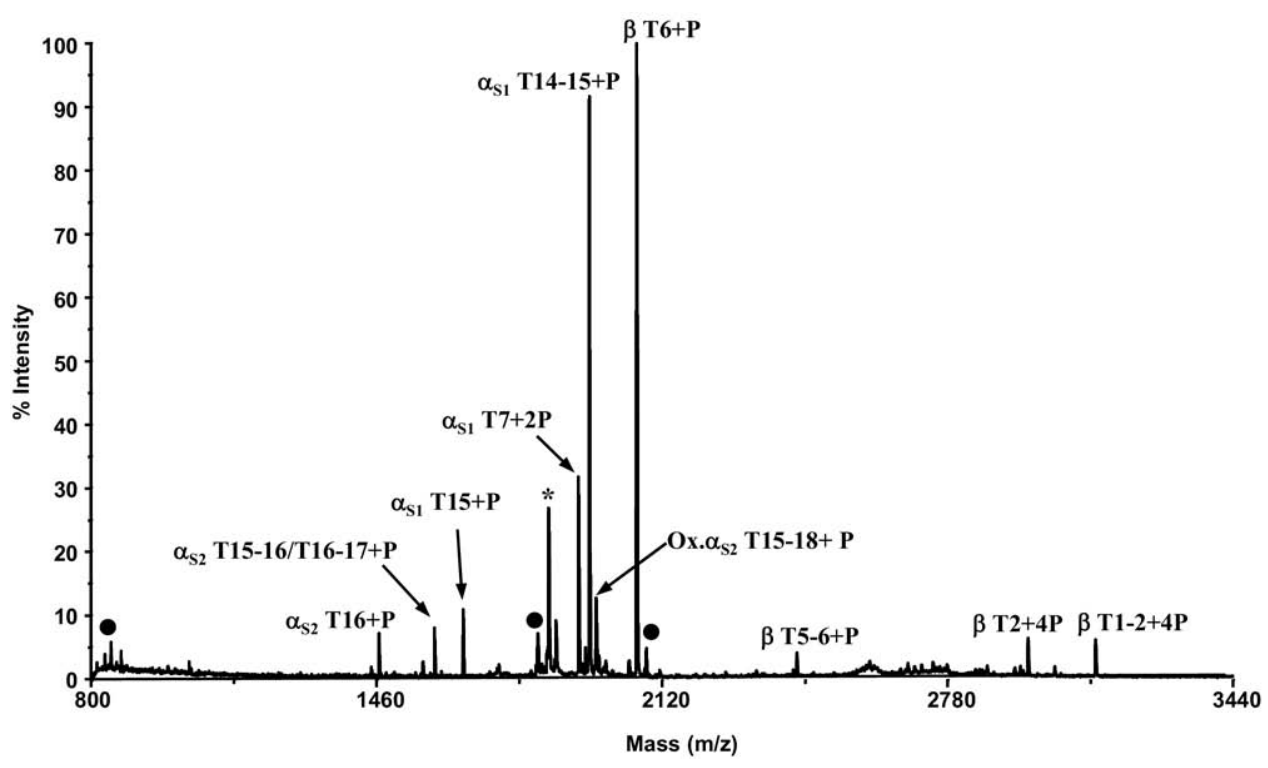

Figure 3. MALDI mass spectrum of an $\alpha$ - and $\beta$-casein tryptic digest resulting from enrichment using magnetic titanium dioxide beads (Phos-Trap kit). The ion labeled with an asterisk corresponds in mass to a previously reported monophosphorylated casein variant [13]. Ions labeled with filled circles represent nonspecifically bound peptides.

dioxide resin NuTip (e.g., $\alpha$-casein S1 T10, $\alpha$-casein S1 T17, and $\beta$-casein T10-11) (Figure $2 \mathrm{~b}$ versus Figure 2a). These results are similar to those reported previously [16] in that zirconium dioxide was observed as being less specific for binding of phosphorylated peptides than titanium dioxide. Comparatively, analyses performed with the mixed resin tip (Figure 2c) showed lower levels of nonspecific binding than that of the zirconium tip (Figure 2b). In our hands, these enrichment results indicate that of the three Glygen NuTips evaluated, the titanium dioxide resin is the most reproducibly selective for phosphopeptides with the least nonspecific binding under the buffer conditions used in these experiments. In addition, the data obtained from the $\mathrm{TiO}_{2}$ NuTip in terms of the number of unique sites of phosphorylation that could be reproducibly enriched in independent experiments are better, in our hands, than those obtained from either the titanium dioxide CRC.

Magnetic titanium beads. Nanoparticles as well as magnetic nanoparticles with a titanium or zirconium surface have also been reported as being selective for enriching phosphorylated peptides. To explore the phosphopeptide enrichment capabilities of a commercially-available magnetic titanium dioxide particle (Perkin Elmer PhosTrap), enrichment of the casein digest mixture was performed using the manufacturer's protocol and the supplied buffers. A MALDI-TOF mass spectrum (Figure 3) acquired from one casein digest eluent of the magnetic titanium resin enrichment experiments contains nine phosphopeptide ions representing nine of the known sites of phosphorylation. The nonspecific binding observed with this resin was minimal and similar to that observed with the other titanium dioxide resins. It should be noted that the chemical background noise observed in the mass range of $\mathrm{m} / \mathrm{z} 2500-2800$ was consistently observed in all eluent analyses from this resin. This most likely inhibited the reproducible observation of several multiply phosphorylated peptides in this region (e.g., $\alpha S 1 \mathrm{~T} 8, \alpha \mathrm{S} 1 \mathrm{~T} 6-7$, and $\alpha \mathrm{S} 2 \mathrm{~T} 2 / \mathrm{T} 1-2)$ (Table 1). Without knowing the composition of the manufacturer's supplied buffers, it is difficult to speculate on the composition of this chemical background. The number of unique sites of phosphorylation observed reproducibly with the Phos-Trap titanium dioxide resin is similar to the Titansphere $\mathrm{TiO}_{2}$ resin and the mixed NuTip resin.

\section{LC/MS/MS Analyses}

We also investigated whether or not additional phosphopeptides could be observed following enrichment using LC/ESI/MS/MS. Because it has been reported $[13,16]$ that analyses of the eluent from the titanium dioxide CRC, which contains sizeable amounts of DHB, is not amenable to LC/MS/MS, the subsequent LC/ MS/MS analyses were performed on the PhosTrap $\mathrm{TiO}_{2}$ eluent and the three different NuTip eluents. For comparison purposes, a tryptic digest of the casein mixture without any enrichment was also analyzed by LC/ MS/MS and structurally diagnostic MS/MS spectra for five phosphopeptides were obtained. These phosphorylated peptides include $\alpha$-casein S1 T15 and T17, $\alpha$ casein S2 T16 and T1-2, and $\beta$-casein T6. For the mixed resin eluent, no additional phosphopeptides were observed by LC/MS/MS. One additional phosphopeptide was observed from a $\mathrm{TiO}_{2}$ NuTip eluent with the se- 
quence ${ }^{112}$ VPNpSAEER ${ }^{119}$ from the S1 variant of $\alpha$-casein LC/MS/MS analysis. This phosphopeptide was the result of an anomalous cleavage.

Conversely, from the LC/MS/MS analyses of the eluents from the Phos-Trap $\mathrm{TiO}_{2}$ resin, two of the phosphopeptides with molecular ions in the mass-tocharge range of 2500-2800, which were observed above the chemical background in only two of the four MALDI/MS analyses were detected by LC/MS/MS. As an example, the ETD MS/MS data of the $[\mathrm{M}+4 \mathrm{H}]^{4+}$ ion of $\mathrm{m} / \mathrm{z} 681.1$ corresponding in mass to the pentaphosphorylated $\alpha$-casein S1 T8 tryptic peptide is shown in Figure 4. In this spectrum, a complement of $\mathrm{c}$ and $\mathrm{z}$ ions are observed due to fragmentations along the peptide backbone chain, which allows the determination of the sites of phosphorylation at Ser 64, 66, 67, 68, and 75. Additionally, CID fragmentation was obtained from the MS/MS analyses of the $[\mathrm{M}+3 \mathrm{H}]^{3+}$ ion of $m / z$ 893.35, which corresponds in mass to the triply phosphorylated $\alpha$-casein S1 T6-7 tryptic peptide (Supplemental Figure S2). These results illustrate the utility of LC in combination with MS/MS for the analyses of complex mixtures and for obtaining greater sequence coverage of proteins.

Under the conditions and MS analyses used in this work, the titanium dioxide NuTip appears to be the most selective phosphopeptide enrichment resin with the least amount of nonspecific binding. Although the nonspecific binding observed with the Titansphere titanium dioxide resin and the mixed resin NuTip was minimal, the overall number of unique sites of phosphorylation observed reproducibly was less than that observed with the $\mathrm{TiO}_{2}$ NuTip. Additionally, our results indicate that the titanium dioxide NuTip resin exhibits a more reproducible phosphopeptide enrichment capability with lower levels of nonspecific binding than the $\mathrm{ZrO}_{2}$ tips. Even though it has been reported that zirconium dioxide preferentially enriches singly phosphorylated peptides and that titanium dioxide preferentially enriches multiphosphorylated peptides [16], we did not observe this trend.

Additional figures of merit. The limits of detection (LOD) for various phosphopeptide enrichment methods have been reported [2, 12, 14, 16, 21] and are important figures of merit when evaluating different media. Inefficient elution from affinity media, incomplete phosphopeptide binding upon loading, and sample loss resulting from sample handling are potential causes of reduced limits of detection. Therefore, determining the minimal amount of digest material required to successfully enrich and detect phosphopeptide sig-

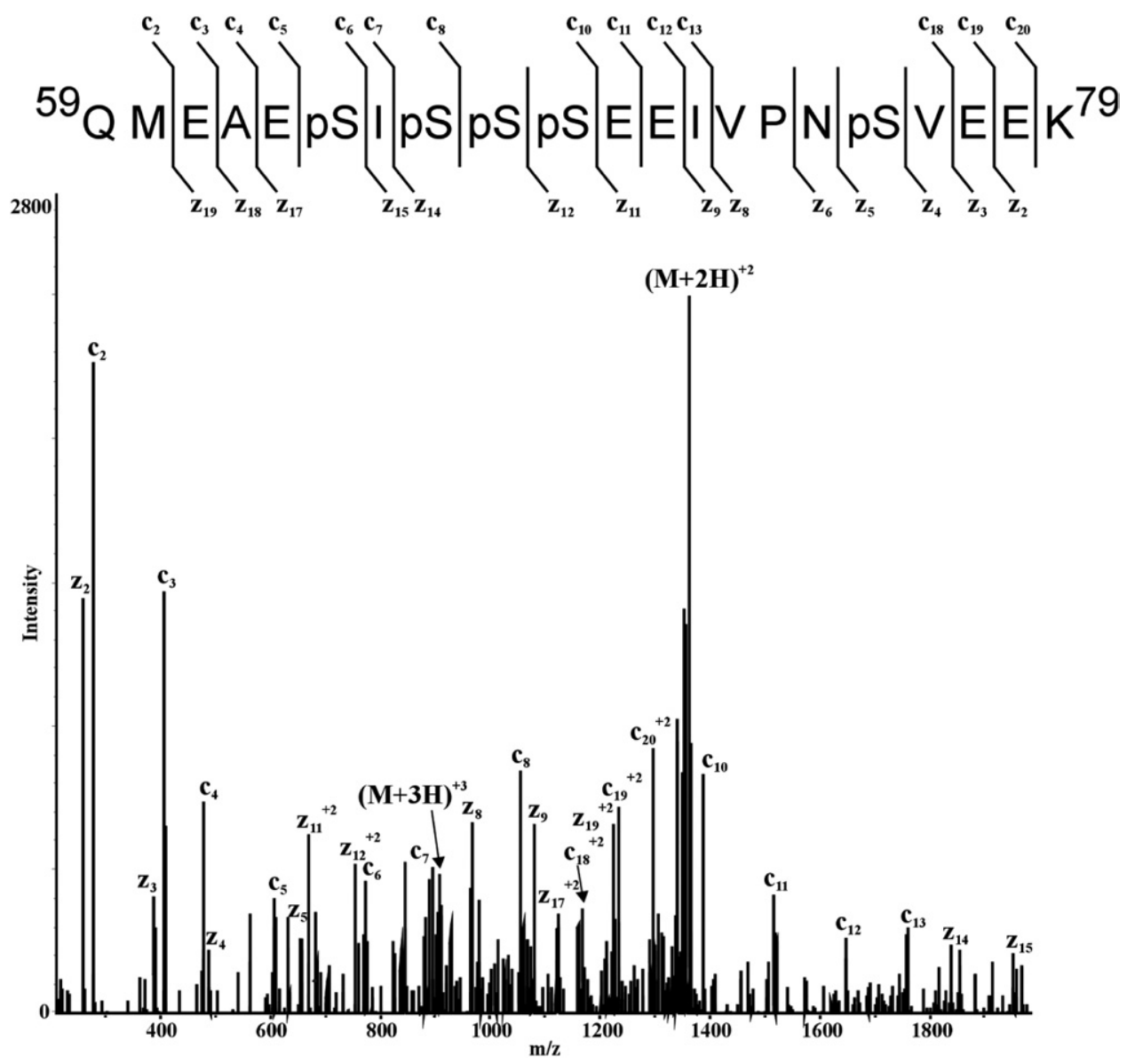

Figure 4. LC/ETD/MS/MS of the $[\mathrm{M}+4 \mathrm{H}]^{4+}$ ion of $\mathrm{m} / \mathrm{z} 681.1$ corresponding in mass to the pentaphosphorylated $\alpha_{\mathrm{S} 1}$ tryptic peptide T8 after enrichment with magnetic $\mathrm{TiO}_{2}$ beads. 
nals is useful. To assess the lowest levels of phosphopeptides from which enrichment could be performed from the casein digest mixtures, independent replicate enrichments of the $\mathrm{TiO}_{2}$ NuTip and the $\mathrm{TiO}_{2}$ PhosTrap resin were evaluated by MALDI/MS. Employing the criteria of a $S / N$ of 3 or greater and the observation of at least two phosphopeptides, the LOD for the $\mathrm{TiO}_{2}$ NuTip was determined to be ca. $125 \mathrm{fmol}$ for $\alpha$-casein and $125 \mathrm{fmol}$ for $\beta$-casein, which showed two monophosphorylated casein peptides, tryptic peptide T6 from $\beta$-casein, and tryptic peptide T14-15 of $\alpha$-casein (S1 variant). The limit of detection for the Phos-Trap kit was determined to be ca. $50 \mathrm{fmol}$ for either $\alpha$ - or $\beta$-casein. The same two monophosphorylated peptides observed with the NuTip were also observed with the magnetic resin. This is a factor of approximately two lower than the limit of detection determined for the titanium NuTip and similar to the LOD reported for the Titansphere titanium dioxide resin [14].

Reproducibility of a given enrichment method can also play a large part in the number of phosphopeptides recovered run-to-run. In our hands, the MOAC resin exhibiting the highest number of phosphorylation sites that could be reproducibly detected by MALDI/MS from four independent enrichment experiments was the $\mathrm{TiO}_{2}$ NuTip (22 sites) followed by the $\mathrm{ZrO}_{2}$ NuTip (17 sites) (Table 1). Reproducibility when using the titanium CRC methodology and the Phos-Trap kit was lower and could be due to several factors. Some variation in reproducibility when using the titanium CRC may be due to loss of resin (and therefore sample) during enrichment due to the small particle size resin and the pore size of the CRC filter. As discussed previously, the chemical noise observed by MALDI/MS in the Phos-Trap eluents likely inhibited reproducible detection of some phosphopeptides.

\section{Application to Avian Histones}

Given the reproducibility results of the NuTip resin and LOD results of the Phos-Trap resin, we chose to further test the capabilities of these two resins by analyzing a more complex biological sample, avian linker and core histones. It is known that histones in avian immature red blood cells undergo many post-translational modifications including phosphorylation, methylation, and acetylation [26-29]. Although the general phosphorylation of chicken histone $\mathrm{H} 1$ has been noted in the literature [27], to our knowledge, only two groups have reported specific sites of phosphorylation of avian histones [27, 30]. Sung and coworkers reported the phosphorylation of Ser-3 and Ser-7 of chicken histone H5 using ${ }^{32} \mathrm{P}$ labeling. Dickman et al. recently published the identification of several modifications of the chicken histones, including phosphorylation of histone $\mathrm{H} 5$ at Ser-3 and Ser-7 and, possibly, at Thr- 1 of this protein. In addition, other sites of phosphorylation that they were unable to unequivocally distinguish include either Ser-22 or Ser- 24 of histone H5 and Ser-1 or Thr-3 of histone H1.11L.

LC/MS/MS analyses of the digested and enriched chicken histone sample identified several phosphorylated peptides (Table 2). As an example, the deconvoluted MS/MS data of the $[\mathrm{M}+2 \mathrm{H}]^{2+}$ ion of $m / z$ 822.2, which corresponds in mass to the N-terminal monophosphorylated acetylated H1.01 tryptic peptide with the sequence SETAPAAAPDAPAPGAK, is shown in Figure 5. [Histone nomenclature is according to Coles et al. [31]]. These MS/MS data show a series of y ions and $\mathrm{b}$ ions which result from fragmentation of the amide bonds in the peptide. In addition, the loss of $\mathrm{H}_{3} \mathrm{PO}_{4}$ from several $b$ ions $\left(b_{7}, b_{8}, b_{11}\right.$, and $\left.b_{13}\right)$ and the loss of water plus $\mathrm{H}_{3} \mathrm{PO}_{4}$ from the $b_{3}$ and $b_{4}$ fragments indicating that the phosphate is located at either serine- 1 or threonine-3. The observation of the ion corresponding in mass to the loss of water and $\mathrm{H}_{3} \mathrm{PO}_{4}$ from the $\mathrm{y}_{16}$ fragment is consistent with a phosphate group located at the Thr- 3 residue versus the Ser- 1 residue. Thus, these data indicate that the site of phosphorylation in this H1.01 tryptic peptide to Thr-3. Collectively, eight phosphopeptides were observed from the histone enrichment and subsequent LC/MS/MS analyses (Table 2, Figure 5, and Supplemental Figures S3-S9). A similar number of, but not identical, phosphopeptides were observed from the two titanium dioxide resins. Therefore, one should utilize more than one enrichment resin

Table 2. Summary of observed phosphorylated peptides following avian histone enrichment

\begin{tabular}{llllclc}
\hline Histone Variant & $\begin{array}{c}\text { Accession } \\
\text { Number }\end{array}$ & $\begin{array}{c}\text { Theoretical } \\
\text { Mass }\end{array}$ & $\begin{array}{c}\text { Observed } \\
\text { Mass }\end{array}$ & Peptide & $\begin{array}{c}\text { Sequence With Modification }{ }^{\text {b }} \\
\text { Phosphorylated } \\
\text { Residue(s) }\end{array}$ \\
\hline \hline H1.10 & AAA48788 & $808.39^{2+}$ & $808.6^{2+}$ & $1-17$ & Ac-SEpTAPAAAPAVAAPAAK & T-3 \\
H1.01 & NP_001035732 & $822.37^{2+}$ & $822.2^{2+}$ & $1-17$ & Ac-SEpTAPAAAPDAPAPGAK & T-3 \\
H1.02 & XP_425456 & $823.40^{+}$ & $823.7^{2+}$ & $1-17$ & Ac-(pS)E(pT)APVAAPAVSAPGAK & S-1 or T-3 \\
H1.11L & NP_001035733 & $991.47^{2+}$ & $991.2^{2+}$ & $1-21$ & Ac-(pS)E(pT)APAPAAEAAPAAAPAPAK & S-1 or T-3 \\
H5 & NP_001038138 & $780.41^{2+}$ & $780.5^{2+}$ & $1-14$ & Ac-(pT)E(pS)LVLSPAPAKPK & T-1 or S-3 \\
H5 & NP_001038138 & $759.40^{2+}$ & $759.47^{2+}$ & $1-14$ & TEpSLVLSPAPAKPK & S-3 \\
H5 & NP_001038138 & $799.39^{2+}$ & $799.2^{2+}$ & $1-14$ & TEpSLVLPSAPAKPK & S-3, S-7 \\
H5 & NP_001038138 & $595.61^{3+}$ & $595.8^{3+}$ & $22-37$ & (pS)A(pS)HPTYSEMIAAAIR & S-22 or S-24 \\
\hline
\end{tabular}

aNomenclature according to reference [31].

${ }^{\mathrm{b}} \mathrm{Ac}=$ acetyl; $\mathrm{p}=$ phosphorylation. 
(a)
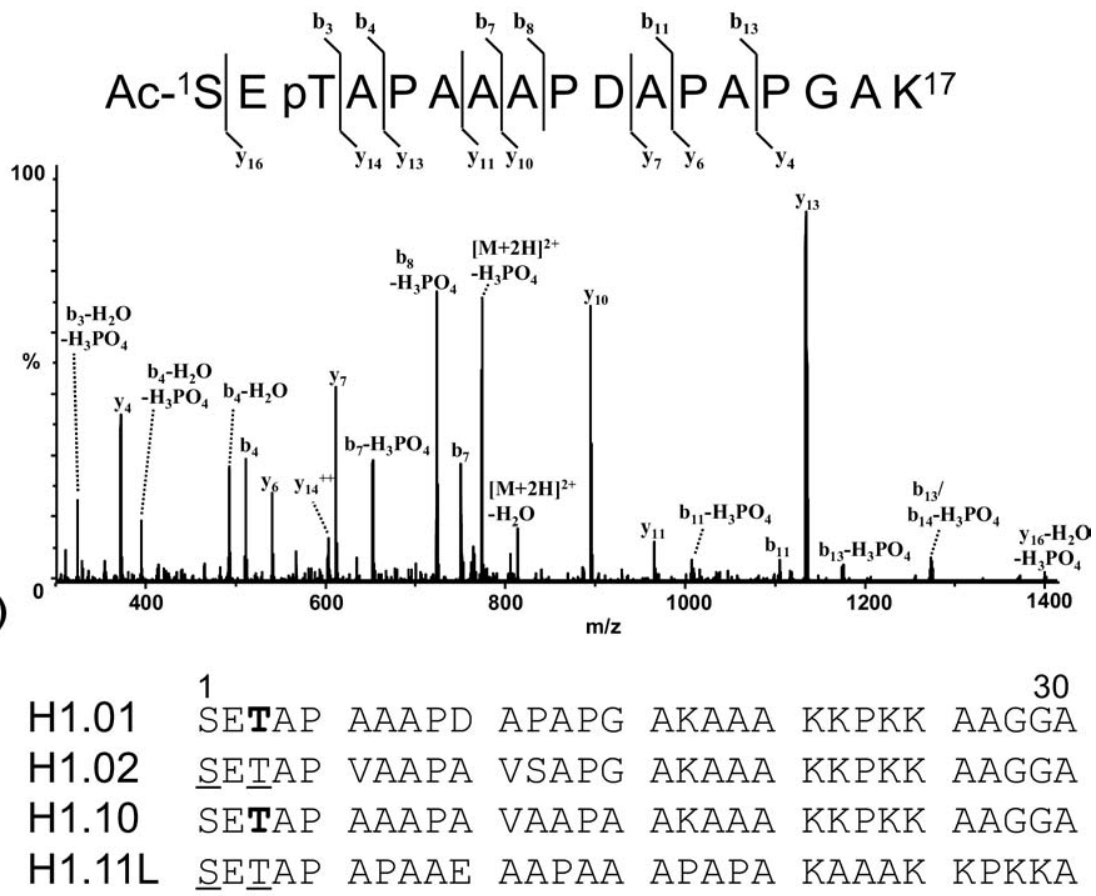

(b) $\mathrm{H} 5$

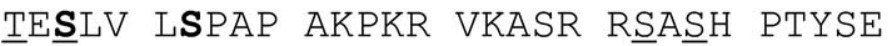

\begin{abstract}
Figure 5. (a) LC/CID/MS/MS of the $(\mathrm{M}+2 \mathrm{H})^{2+}$ ion of $\mathrm{m} / \mathrm{z} 822.2$ corresponding in mass to the $\mathrm{N}$-terminal monophosphorylated acetylated histone H1.01 tryptic peptide. (b) N-terminal 30 residues of avian histone proteins for which sites of phosphorylation were determined. Amino acids in bold indicate residues in which a site of phosphorylation was confirmed. Underlined amino acids indicate residues in which the site of phosphorylation could not be unequivocally assigned.
\end{abstract}

and optimize the conditions according to sample volume and complexity.

From our MS analyses of phospho-enriched samples, we were able to identify previously unreported sites of phosphorylation. These include Thr-3 of histone H1.01 (Figure 5a) and Thr-3 of histone H1.10 (Supplemental Figure S3). Phosphorylation of histone H1.02 at Ser-1 or Thr-3 was also observed, but could not be unambiguously identified (Supplemental Figure S4). In addition to these novel sites of phosphorylation, the additional sites of phosphorylation of histone H5 (Ser-3, Ser-7, possibly Thr-1, and Ser-22 or Ser-24) and of histone H1.11L (Ser-1 or Thr-3) were identified (Supplemental Figures S5-S9). The $\mathrm{N}$-terminal 30 residues of the chicken histones for which sites of phosphorylation were determined are shown in Figure 5b. Residues shown in bold indicate an amino acid for which a site of phosphorylation was confirmed. Underlined amino acids indicate residues in phosphopeptides for which the site of phosphorylation could not be unequivocally assigned. Of note, only the phosphorylation site at Ser-3 of histone H5 was observed by LC/MS/MS without enrichment. Several of the sites of phosphorylation determined for the chicken histone $\mathrm{H} 1$ proteins are homologous to sites of phosphorylation determined for histone $\mathrm{H} 1$ proteins from mammals [32, 33].

\section{Conclusion}

All titanium dioxide resins evaluated in this study are capable of enriching phosphopeptides from a casein digest sample, albeit to different extents. These data demonstrate the importance of the experimental conditions employed for the enrichment techniques. Similar to previous reports, organic content in binding and washing solutions proved to be beneficial for the metal oxide resins. Both MALDI and ESI-MS analyses of the titanium dioxide NuTip eluents resulted in the reproducible observation of a greater number of unique sites of phosphorylation with the least amount of nonspecific binding compared with the other MOAC resins. These results demonstrate that enrichment procedures before MS analyses dramatically improve detection and sequencing of phosphopeptides compared with analyses without enrichment. In addition, enrichment reduces sample complexity, thereby, reducing the co-elution of peptides during LC/MS/MS analyses.

The applicability of phosphopeptide enrichment of a biological sample was illustrated using titanium dioxide enrichment media. For the avian histone tryptic digest, eight phosphorylated tryptic peptides were observed following enrichment and subsequent LC/ MS/MS analyses. Seven of the eight phosphopeptides were not observed without titanium dioxide enrich- 
ment. From these analyses, four sites of phosphorylation were unequivocally determined; two of which have not been reported previously (i.e., Thr-3 of histone H1.10 and Thr-3 of histone H1.01). Four additional phosphopeptides were observed, however, the site of phosphorylation could not be distinguished, but was localized to one of two possible amino acids.

In summary, two of the titanium dioxide resins showed greater selectivity and sensitivity in our hands, however, these analyses were performed on samples of limited complexity. Therefore, depending on sample volume and complexity, it is important to optimize enrichment procedures accordingly. These methods should aid in the investigation of proteins post-translationally modified with phosphate, especially those present at low concentrations as was demonstrated by successful enrichment at the femtomole level.

\section{Acknowledgments}

The authors acknowledge support for this research by the Intramural Research Program of the NIH, National Institute of Environmental Health Sciences (ES050171). The authors thank Dr. Roxana Iacob and Dr. Jeffrey Kuhn for their assistance and thoughtful input, and Dr. Allison Schorzman and Dr. Jason Williams for critical review of this manuscript. NIEHS does not endorse or recommend any commercial products, processes, or services. The views and opinions expressed by authors affiliated with NIEHS do not necessarily state or reflect those of the U.S. Government, and they may not be used for advertising or product endorsement purposes.

\section{Appendix A Supplementary Material}

Supplementary material associated with this article may be found in the online version at doi:10.1016/ j.jasms.2010.06.005.

\section{References}

1. McLachlin, D. T.; Chait, B. T. Analysis of Phosphorylated Proteins and Peptides by Mass Spectrometry. Curr. Opin. Chem. Biol. 2001, 5, 591-602.

2. Reinders, J.; Sickmann, A. State-of-the-Art in Phosphoproteomics. Proteomics 2005, 5, 4052-4061.

3. Thingholm, T. E.; Jensen, O. N.; Larsen, M. R. Analytical Strategies for Phosphoproteomics. Proteomics 2009, 9, 1451-1468.

4. Sun, X.; Chiu, J. F.; He, Q. Y. Application of Immobilized Metal Affinity Chromatography in Proteomics. Expert Rev. Proteom. 2005, 2, 649-657.

5. Dunn, J. D.; Reid, G. E.; Bruening, M. L. Techniques for Phosphopeptide Enrichment Prior to Analysis by Mass Spectrometry. Mass Spectrom. Rev. 2010, 29, 29-54.

6. Thingholm, T. E.; Larsen, M. R. The Use of Titanium Dioxide MicroColumns to Selectively Isolate Phosphopeptides from Proteolytic Digests. Methods Mol. Biol. 2009, 527, 57-55.

7. Zhou, W.; Merrick, B. A.; Khaledi, M. G.; Tomer, K. B. Detection and Sequencing of Phosphopeptides Affinity Bound to Immobilized Metal Ion Beads by Matrix-Assisted Laser Desorption/Ionization Mass Spectrometry. J. Am. Soc. Mass Spectrom. 2000, 11, 273-282.

8. Merrick, B. A.; Zhou, W.; Martin, K. J.; Jeyarajah S.; Parker, C. E.; Selkirk, J. K.; Tomer, K. B.; Borchers, C. H. Site-Specific Phosphorylation of Human p53 Protein Determined by Mass Spectrometry. Biochemistry 2001, 40, 4053-4066.

9. Deterding, L. J.; Cutalo, J. M.; Tomer, K. B. Proteomic Methods for Phosphorylation Site Mapping In Proteins and Proteomics: A Laboratory Manual; Simpson, R., Ed.; Cold Spring Harbor Laboratory Press: Cold Spring Harbor, NY, 2003; p. 597.
10. Cao, H.; Deterding, L. J.; Venable, J. D.; Kennington, E. A.; Yates, J. R.; Tomer, K. B.; Blackshear, P. J. Identification of the Anti-Inflammatory Protein Tristetraprolin as a Hyperphosphorylated Protein by Mass Spectrometry and Site-Directed Mutagenesis. Biochem. J. 2006, 394, 285-297.

11. Connor, P. A.; McQuillan, A. J. Phosphate Adsorption onto $\mathrm{TiO}_{2}$ from Aqueous Solutions: An In Situ Internal Reflection Infrared Spectroscopic Study. Langmuir 1999, 15, 2916-2921.

12. Pinkse, M. W.; Uitto, P. M.; Hilhorst, M. J.; Ooms, B.; Heck, A. J. Selective Isolation at the Femtomole Level of Phosphopeptides from Proteolytic Digests Using 2D-NanoLC-ESI-MS/MS and Titanium Oxide Precolumns. Anal. Chem. 2004, 76, 3935-3943.

13. Larsen, M. R.; Thingholm, T. E.; Jensen O. N.; Roepstorff, P.; Jorgensen, T. J. D. Highly Selective Enrichment of Phosphorylated Peptides from Peptide Mixtures Using Titanium Dioxide Microcolumns. Mol. Cell. Proteom. 2005, 4, 873-886.

14. Thingholm, T. E.; Jorgensen, T. J.; Jensen O. N.; Larsen, M. R. Highly Selective Enrichment of Phosphorylated Peptides using Titanium Dioxide. Nat. Protoc. 2006, 1, 1929-1935.

15. Kweon, H. K.; Håkansson, K. Selective Zirconium Dioxide-Based Enrichment of Phosphorylated Peptides for Mass Spectrometric Analysis. Anal. Chem. 2006, 78, 1743-1749.

16. Jensen, S. S.; Larsen, M. R. Evaluation of the Impact of Some Experimental Procedures on Different Phosphopeptide Enrichment Techniques. Rapid Commun. Mass Spectrom. 2007, 21, 3635-3645.

17. Liang, X.; Fonnum, G.; Hajivandi, M.; Stene, T.; Kjus, N. H.; Ragnhildstveit, E.; Amshey, J. W.; Predki, P.; PopeM. Quantitative Comparison of IMAC and $\mathrm{TiO}_{2}$ Surfaces Used in the Study of Regulated, Dynamic Protein Phosphorylation. J. Am. Soc. Mass Spectrom. 2007, 18, 1932-1944.

18. Kweon, H. K.; Håkansson, K. Metal Oxide-Based Enrichment Combined with Gas-Phase Ion-Electron Reactions for Improved Mass Spectrometric Characterization of Protein Phosphorylation. J. Proteome Res. 2008, 7, 749-755.

19. Simon, E. S.; Young, M.; Chan, A.; Bao, Z. Q.; Andrews, P. C. Improved Enrichment Strategies for Phosphorylated Peptides on Titanium Dioxide using Methyl Esterification and pH Gradient Elution. Anal. Biochem. 2008, 377, 234-242

20. Yu, Y.-Q.; Fournier, J.; Gilar, M.; Gebmler, J. C. Phosphopeptide Enrichment using Microscale Titanium Dioxide Solid Phase Extraction. J. Sep. Sci. 2009, 32, 1189-1199.

21. Aryal, U. K.; Ross, A. R. S. Enrichment and Analysis of Phosphopeptides Under Different Experimental Conditions using Titanium Dioxide Affinity Chromatography and Mass Spectrometry. Rapid Commun. Mass Spectrom. 2010, 24, 219-231.

22. Bai, Z.; Liu, B.; Li, W.; Li, P.; Wang, H.; Wang, H. The Development of an Improved Simple Titanium Dioxide Enrichment Method for Phosphoproteomic Research. Rapid Commun. Mass Spectrom. 2009, 23, 30133017.

23. Schmidt, A.; Csaszar, E.; Ammerer, G.; Mechtler, K. Enhanced Detection and Identification of Multiply Phosphorylated Peptides using $\mathrm{TiO}_{2}$ Enrichment in Combination with MALDI TOF/TOF MS. Proteomics 2008, 8, 4577-4592.

24. Lee, D. H.; McComb, M. E.; Bromirski, M.; Jilkine, A.; Ens, W.; Standing, K. G.; Perreault, H. On-Membrane Digestion of $\beta$-casein for Determination of Phosphorylation Sites by Matrix-Assisted Laser Desorption/ Ionization Quadrupole/Time-of-Flight Mass Spectrometry. Rapid Commun. Mass Spectrom. 2001, 15, 191-202.

25. Janek, K.; Wenschuh, H.; Bienert, M.; Krause, E. Phosphopeptide Analysis by Positive and Negative Ion Matrix-Assisted Laser Desorption/Ionization Mass Spectrometry. Rapid Commun. Mass Spectrom. 2001, 15, 1593-1599.

26. Sung, M. T.; Harford, J.; Bundman, M.; Vidalakas, G. Metabolism of Histones in Avian Erythroid Cells. Biochemistry 1977, 16, 279-285.

27. Sung, M. T.; Freedlander, E. F. Sites of In Vivo Phosphorylation of Histone H5. Biochemistry 1978, 17, 1884-1890.

28. Sung, M. T. Phosphorylation and Dephosphorylation of Histone V (H5): Controlled Condensation of Avian Erythrocyte Chromatin. Biochemistry 1977, 16, 286-290.

29. Pikaart, M.; Irving, J.; Villeponteau, B. Decline in Histone H5 Phosphorylation During Erythroid Senescence in Chick Embryos. Mech. Ageing Dev. 1991, 59, 189-195.

30. Snijders, A. P. L.; Pongdam, S.; Lambert, S. J.; Wood, C. M.; Baldwin J. P.; Dickman, M. J. Characterization of Post-Translational Modifications of the Linker Histones H1 and H5 from Chicken Erythrocytes Using Mass Spectrometry. J. Proteome Res. 2008, 7, 4326-4335.

31. Coles, L. S.; Robins, A. J.; Madley, L. K.; Wells, J. R. E. Characterization of the Chicken Histone H1 Gene Complement. Generation of a Complete Set of Vertebrate H1 Protein Sequences. J. Biol. Chem. 1987, 262, 9656-9663.

32. Garcia, B. A.; Busby, S. A.; Barber, C. M.; Shabanowitz, J.; Allis, C. D. Hunt, D. F. Characterization of Phosphorylation Sites on Histone H1 Isoforms by Tandem Mass Spectrometry. J. Proteome Res. 2004, 3, 1219-1227.

33. Wisniewski, J. R.; Zougman, A.; Kruger, S.; Mann, M. Mass Spectrometric Mapping of the Linker Histone H1 Variants Reveals Multiple Acetylations, Methylations, and Phosphorylations as Well as Differences Between Cell Culture and Tissue. Mol. Cell. Proteom. 2007, 6, 72-87. 